\title{
BOUNDARIES AND MODULAR IDEALS ON LOCALLY COMPACT GROUPS
}

\author{
WILLIAM MORAN AND GEORGE A. WILLIS
}

(Communicated by Palle E. T. Jorgensen)

\begin{abstract}
The purpose of this paper is to exhibit an example of a modular ideal of the form $L^{1}(G) *(\delta(e)-\mu)$, where $\mu$ is a probability measure on the locally compact group $G$ which has all of its convolution powers purely singular.
\end{abstract}

\section{INTRODUCTION}

The aim of this paper is to present an example in the theory of the Poisson boundaries of stationary random walks on locally compact groups. There are various formalisations of the concept of a Poisson boundary. An account of the standard theory can be found in [4], though only in the context of discrete groups. It is appropriate here to use an alternative view of boundaries presented by Willis in [5], and which we describe briefly. Let $G$ be a locally compact group and $\mu$ a probability measure on $G$ which is to be thought of as the distribution associated with the random walk. It is shown in [5] that the closed left ideal $J_{\mu}=c l\left(L^{1}(G) \star(\delta(e)-\mu)\right)$ has the property that $L^{1}(G) / J_{\mu}$ is an abstract Lspace and so, by Kakutani's Theorem, of the form $L^{1}(\Omega, \sigma)$ for some measure $\sigma$ on the measurable space $\Omega$. A $G$-action may be introduced on $\Omega$ that makes $\sigma$ into a quasi-invariant measure. There is also a $\mu$-stationary measure; that is, a probability measure $\nu$ on $\Omega$ satisfying $\int g \cdot \nu d \mu(g)=\nu$, where $g \cdot \nu$ indicates the action on the measure $\nu$ induced by the action of $g \in G$ on the underlying space. Both the measure class of $\sigma$ and the probability measure $\nu$ are uniquely determined by the ideal $J_{\mu}$. The collection consisting of the $G$-space $\Omega$, the quasi-invariant measure $\sigma$ and the $\mu$-stationary measure $\nu$ is called the Poisson boundary of the random walk associated with $\mu$.

The relationship between the two measures $\sigma$ and $\nu$ on $\Omega$ is particularly important and interesting. It is known, for instance, that when $\mu$ is absolutely continuous and nondegenerate (that is, the smallest closed semigroup containing the support of $\mu$ is $G$ itself) then the two measures are equivalent([5]). It is

Received by the editors January 21, 1990.

1980 Mathematics Subject Classification (1985 Revision). Primary 43A05, 43A20, 60B15, 60J15, $60 \mathrm{~J} 50$. 
also known that under the weaker condition of absolute continuity of $\mu, \nu$ itself is absolutely continuous with respect to $\sigma$. However the converse fails as the example we shall give demonstrates.

To explore this circle of ideas further, and explain the particular relevance of our example, we need to recall the concept of a modular ideal. A left ideal $I$ in $L^{1}(G)$ is modular if there exists $u \in L^{1}(G)$ such that $f-f \star u$ belongs to $I$ for every $f \in L^{1}(G)$. The element $u$ is referred to as a right modular unit for the ideal $I$. Now $J_{\mu}$ is modular if and only if $\nu$ is absolutely continuous with respect to $\sigma$. One proves this by observing that $u+J_{\mu}$ maps to $\nu$ under the isomorphism between $L^{1}(G) / J_{\mu}$ and $L^{1}(\Omega, \sigma)$. (Cf. [5, Propositions 2.4 and 2.5].) Evidently, if $\mu$ is absolutely continuous then $J_{\mu}$ is modular. There are, however, weaker conditions which guarantee the modularity of $J_{\mu}$; for example, if some convolution power of $\mu$ is not singular with respect to Haar measure then in this case also $J_{\mu}$ is modular. On the other hand, this is not really a new situation because it is relatively straightforward to see that under these circumstances there is another measure $\mu^{\prime}$ absolutely continuous with respect to Haar measure such that $J_{\mu}=J_{\mu^{\prime}}$. Let us agree to say that a closed left ideal $J$ in $L^{1}(G)$ is absolutely continuous if it is of the form $J_{\mu}$ for some measure $\mu$ which is absolutely continuous. The example we produce has the property that the ideal $J_{\mu}$ is modular but not absolutely continuous.

The groups we are interested in, and on which our example is set, are semidirect products of $\mathbf{Z}$ with another abelian group $H$, with the action of $\mathbf{Z}$ on $H$ generated by an automorphism with specified properties. It is of interest and appropriate to develop the theory we need in the context of general groups of this nature before fixing on a specific example.

\section{BASIC RESULTS}

We refer to [5] for the unexplained terminology and results. Let $H$ be a locally compact abelian group and let $\alpha$ be an automorphism of $H$. We write $\hat{\alpha}$ for the adjoint automorphism on the dual group $\widehat{H}$, and $\alpha^{*}$ for the map between the spaces of measures induced by $\alpha$. The group $G$ will be the semidirect product of $\mathbf{Z}$ with $H$ where the action of $\mathbf{Z}$ on $H$ is given by the automorphism $\alpha$. Thus, as a topological space, $G$ is just $\mathbf{Z} \times H$ and the multiplication formula is:

$$
(m, h) \cdot(n, k)=\left(m+n, \alpha^{-n}(h)+k\right) .
$$

The automorphism $\alpha$ will be assumed to have the property that the function

$$
\phi(a, \gamma)=\sum_{n=1}^{\infty}\left|\gamma\left(\alpha^{n}(a)\right)-1\right|
$$

is bounded on compact sets of $H \times \widehat{H}$. It will be convenient to change notation and refer to the probability measure which is the distribution of the random walk as $\mu^{\prime}$. We shall choose $\mu^{\prime}$ to be supported on $\{1\} \times H$ and to have compact 
support. Write $\mu$ for the measure $\mu^{\prime}$ when thought of as sitting on $H$, so that $\mu^{\prime}=\mu \star \delta(1,0)$. We shall assume throughout that the closed subgroup generated by the support of $\mu^{\prime}$ is the whole of $G$. Under these conditions it is possible to calculate the boundary associated with $\mu^{\prime}$.

Proposition 1. $L^{1}(G) / J_{\mu^{\prime}}$ is isometrically isomorphic to $L^{1}(H)$ as an $L^{1}(G)$ module. In other words, the boundary of $\mu^{\prime}$ may be taken to be $H$ which is a $G$-space under the action $(m, h) . k=\alpha^{-m}(h+k)$. Moreover the $\mu^{\prime}$-stationary measure $\nu$ on $H$ is the infinite convolution product $\star_{n=0}^{\infty}\left(\alpha^{*}\right)^{n}(\mu)$, and an appropriate choice of the quasi-invariant measure $\sigma$ is Haar measure on $H$.

Before proving this result, we need to establish the existence of the infinite convolution product. This is done in the next lemma.

Lemma 1. The infinite convolution product $\star_{n=0}^{\infty}\left(\alpha^{*}\right)^{n}(\mu)$ converges to a probability measure $\nu$ in the strong operator topology on $L^{1}(H)$.

Proof. First we note that the support of this proposed measure is compact. If the measure exists, then its support is the closure $D$ of $\bigcup_{n=0}^{\infty} \alpha^{n}(K)$ where $K$ is the support of $\mu$. We show that $D$ is precompact in $H$. A neighbourhood of the identity in $H$ contains one of the form

$$
N=\{h:|\gamma(h)-1|<\epsilon \quad \gamma \in K\},
$$

where $\mathrm{K}$ is a compact subset of $\widehat{H}$, so that because of the property (1) we are assuming for $\alpha$ it is clear that $\bigcup_{n=n_{0}}^{\infty} \alpha^{n}(K)$ is contained in $N$ for some large enough $n_{0}$. Since the finite union $\bigcup_{n=0}^{n_{0}-1} \alpha^{n}(K)$ is compact, it can be covered by finitely many translates of $N$. Therefore $D$ is compact. Now we observe that $\nu_{m}=\star_{n=0}^{m}\left(\alpha^{*}\right)^{n}(\mu)$ converges weak- $*$ to a probability measure $\nu$. This follows from (1) also, since for $\gamma \in \widehat{H}, \sum_{n=0}^{\infty}\left|\gamma\left(\alpha^{n}(t)\right)-1\right|<\infty$ and the sum is bounded on $K$. To obtain strong operator convergence, note that if $D_{m}$ denotes $\bigcup_{n=m}^{\infty} \alpha^{n}(K)$, then by $(1),\left(D_{n}\right)$ is a sequence of compact sets converging to the identity in $H$. Therefore, for $f \in L^{1}(H)$, and $n \geq m$,

$$
\left\|\nu_{n} \star f-\nu_{m} \star f\right\| \leq\left\|\star_{k=m+1}^{n}\left(\alpha^{*}\right)^{n}(\mu) \star f-f\right\| \rightarrow 0
$$

as $n, m \rightarrow \infty$.

Proof of Proposition 1. Observe first that $\nu$ is $\mu^{\prime}$-stationary. This is the case because

$\mu^{\prime} \cdot \nu=(\mu \star \delta(1,0)) \cdot \nu=\mu \star(\delta(1,0) \cdot \nu)=\mu \star \alpha^{*}(\nu)=\mu \star \star_{n=1}^{\infty}\left(\alpha^{*}\right)^{n}(\mu)=\nu$.

It follows that if $f$ belongs to $J_{\mu^{\prime}}$, then $f \cdot \nu=0$, and so we are able to define a linear map $T: L^{1}(G) / J_{\mu^{\prime}} \rightarrow L^{1}(H)$ by

$$
T\left(f+J_{\mu^{\prime}}\right)=f \cdot \nu .
$$


We can write $f$ as a sum $\sum_{n \in \mathbf{Z}} f_{n} \star \delta(n, 0)$ where $f_{n}$ is in $L^{1}(H)$ so that

$$
T\left(f+J_{\mu^{\prime}}\right)=\sum_{n \in \mathbf{Z}} f_{n} \star\left(\alpha^{*}\right)^{n}(\nu) .
$$

It is now clear that $T$ is well-defined and is an $L^{1}(G)$-homomorphism from $L^{1}(G)$ to $L^{1}(H)$. Since $\nu$ is a probability measure, $\left\|T\left(f+J_{\mu^{\prime}}\right)\right\| \leq\left\|f+J_{\mu^{\prime}}\right\|$ for $f \in L^{1}(G)$. Next we prove that the reverse inequality holds. To do this, fix $f$ in $L^{1}(G)$ and choose $\epsilon>0$.

Note that

$$
\mu^{\prime n}=\star_{n=0}^{n-1}\left(\alpha^{*}\right)^{i}(\mu) \star \delta(n, 0),
$$

and consequently,

$$
\begin{aligned}
\left\|f \star \frac{1}{N} \sum_{n=N+1}^{2 N}\left(\mu^{\prime}\right)^{n}\right\| & =\left\|\frac{1}{N} \sum_{n=N+1}^{2 N}\left(f \star\left(\star_{i=0}^{n-1}\left(\alpha^{*}\right)^{i}(\mu)\right) \star \delta(n, 0)\right)\right\| \\
& =\left\|\frac{1}{N} \sum_{n=N+1}^{2 N} \sum_{k \in \mathbf{Z}}\left(f_{k} \star\left(\star_{i=0}^{n-1}\left(\alpha^{*}\right)^{(i+k)}(\mu)\right) \star \delta(n+k, 0)\right)\right\| \\
& \leq\left\|\frac{1}{N} \sum_{n=N+1}^{2 N} \sum_{k \in \mathbf{Z}}\left(f_{k} \star\left(\alpha^{*}\right)^{k}(\nu) \star \delta(n+k, 0)\right)\right\|+\epsilon
\end{aligned}
$$

for $N$ sufficiently large. Here we have used the fact that, by Lemma 1, $\star_{i=0}^{n-1}\left(\alpha^{*}\right)^{(i+k)}(\mu)$ converges to $\left(\alpha^{*}\right)^{k}(\nu)$ in the strong operator topology. Now

$$
\begin{aligned}
\| \frac{1}{N} & \sum_{n=N+1}^{2 N}\left(\sum_{k \in \mathbf{Z}} f_{k} \star\left(\alpha^{*}\right)^{k}(\nu) \star \delta(n+k, 0)\right) \| \\
= & \left\|\sum_{k \in \mathbf{Z}}\left(f_{k} \star\left(\alpha^{*}\right)^{k}(\nu) \star \delta(k, 0)\right) \star \frac{1}{N} \sum_{n=N+1}^{2 N} \delta(n, 0)\right\| \\
& \rightarrow\left\|\left(\sum_{k \in \mathbf{Z}} f_{k} \star\left(\alpha^{*}\right)^{k}(\nu)\right)\right\|=\left\|T\left(f+J_{\mu^{\prime}}\right)\right\|
\end{aligned}
$$

as $N \rightarrow \infty$. Therefore,

$$
\left\|f+J_{\mu^{\prime}}\right\| \leq\left\|f \star \frac{1}{N} \sum_{n=N+1}^{2 N}\left(\mu^{\prime}\right)^{n}\right\| \leq\left\|T\left(f+J_{\mu^{\prime}}\right)\right\|+\epsilon,
$$

for sufficiently large $N$. This gives the required conclusion.

Because of (1) the support of $\left(\alpha^{*}\right)^{n}(\nu)$ is a compact set approaching the identity, accordingly, $\left\|f-f \star\left(\alpha^{*}\right)^{n}(\nu)\right\| \rightarrow 0$ as $n \rightarrow \infty$ for $f \in L^{1}(H)$. Now

$$
T\left(f \star \delta(n, 0)+J_{\mu^{\prime}}\right)=f \star\left(\delta(n, 0) \cdot \nu=f \star\left(\alpha^{*}\right)^{n}(\nu) \rightarrow f\right.
$$

as $n \rightarrow \infty$. Since $T$ is an isometry, its image is closed, and so $T$ is surjective. We have thus shown that $L^{1}(G) / J_{\mu^{\prime}}$ is isometrically isomorphic to $L^{1}(H)$. The remainder of the proof is straightforward. 
It is now relatively straightforward to characterise the modular ideals among the $J_{\mu^{\prime}}$ in terms of the stationary measure $\nu$.

Proposition 2. The ideal $J_{\mu^{\prime}}$ is modular if and only if $\nu$ is absolutely continuous with respect to Haar measure on $H$.

Proof. Suppose first that $\nu$ is absolutely continuous with respect to Haar measure. Then $\nu$ belongs to $L^{1}(H)$ and so there is a function $u$ in $L^{1}(G)$ such that $\nu=T\left(u+J_{\mu^{\prime}}\right)=u \cdot \nu$. Now for $f$ in $L^{1}(G)$,

$$
(f-f \star u) \cdot \nu=f \cdot \nu-f \cdot(u \cdot \nu)=0,
$$

and so, by Proposition 1, $f-f \star u$ belongs to $J_{\mu^{\prime}}$. Therefore $J_{\mu^{\prime}}$ is modular.

Conversely, if $J_{\mu^{\prime}}$ is modular with unit $u$ then $T\left(u+J_{\mu^{\prime}}\right)=u \cdot \nu$ which belongs to $L^{1}(H)$. For $f \in L^{1}(H) \subset L^{1}(G), f \star(\delta(e)-u)$ belongs to $J_{\mu^{\prime}}$ and so

$$
0=(f \star(\delta(e)-u)) \cdot \nu=f \star(\nu-u \cdot \nu) .
$$

Hence $\nu=u \cdot \nu$ and since $u \cdot \nu$ belongs to $L^{1}(H), \nu$ is absolutely continuous.

We shall say that a measure on $H$ is lower semicontinuous if it is absolutely continuous with respect to Haar measure and some representative of its RadonNikodym derivative is lower semicontinuous. The example we seek has the property that $J_{\mu^{\prime}}$ is modular but not absolutely continuous, that is, not of the form $J_{\mu^{\prime \prime}}$ for $\mu^{\prime \prime}$ absolutely continuous. Evidently, we shall need a criterion for determining whether $\mu^{\prime \prime}$ is absolutely continuous expressed in terms of the stationary measure $\nu$. This is provided by the concept of lower semicontinuous measure and the next result.

Proposition 3. Let $\mu^{\prime}$ be as in Proposition 1. Then $J_{\mu^{\prime}}$ is absolutely continuous if and only if $\nu$ is lower semi-continuous.

Proof. If there is an absolutely continuous measure $\mu^{\prime \prime}$ such that $J_{\mu^{\prime}}=J_{\mu^{\prime \prime}}$ then $J_{\mu^{\prime}}$ is modular (with modular unit $\mu^{\prime \prime}$ ) and so, by Proposition 2, $\nu$ is absolutely continuous. In this case let $f$ be the Radon-Nikodym derivative of $\nu$ with respect to Haar measure and write $\mu^{\prime \prime}=\sum_{n \in \mathbf{Z}} \mu_{n} \star \delta(n, 0)$ where $\mu_{n}$ is a positive measure on $H$. For each $N$ let $\nu_{N}$ be the positive measure on $H$ whose Radon-Nikodym derivative is $\phi_{N}=\min (f, N)$, and let $\mu_{N}^{\prime \prime}=$ $\sum_{|n|<N} \mu_{n} \star \delta(n, 0)$. Then $\left(\nu_{N}\right)_{N=1}^{\infty}$ and $\left(\mu_{N}^{\prime \prime}\right)_{N=1}^{\infty}$ are increasing sequences of positive measures with limits $\nu$ and $\mu^{\prime \prime}$ respectively. It follows that $\mu_{N}^{\prime \prime}$. $\left.\nu_{N}\right)_{N=1}^{\infty}$ is an increasing sequence of positive measures with limit $\mu^{\prime \prime} \cdot \nu=\nu$. Now for each $\mathrm{n},\left(\mu_{n} \star \delta(n, 0)\right) \cdot \nu_{N}=\mu_{n} \star\left(\alpha^{*}\right)^{n}\left(\nu_{N}\right)$, which is the product of two absolutely continuous measures one of which has bounded Radon-Nikodym derivative. The resulting measure must then have a continuous Radon-Nikodym derivative and so the Radon-Nikodym derivative $f$ of $\nu$ is the limit of an 
increasing sequence of continuous functions, in other words $\nu$ is lower semicontinuous.

For the converse, assume that $\nu$ is lower semicontinuous, and suppose that we have found a positive measure, $\lambda$ on $G$ such that

$$
\lambda \cdot \nu \leq \nu, \quad(\lambda \cdot \nu)(H)>(1-\delta) \nu(H)=1-\delta
$$

for some $\delta>0$, and the Radon-Nikodym derivative of $\lambda \cdot \nu$ with respect to $m$ is continuous (that is, has a representative which is continuous). We shall show that there is a positive measure $\lambda^{\prime}$ on $G$ with $\lambda^{\prime} \geq \lambda$ which satisfies the above conditions with $\delta$ replaced by $\delta / 2$. It is clear how the the proof of the result may be completed from this.

Since $\lambda \cdot \nu$ is continuous and $\lambda \cdot \nu \leq \nu, \nu-\lambda \cdot \nu$ is lower semicontinuous and nonnegative. Let its Radon-Nikodym derivative with respect to $m$ be $f$. Then $f$ is lower semicontinuous, and as a result we can find a continuous function $h$ whose support is compact and entirely contained in $U=\{t: f(t)>0\}$ and which itself satisfies

$$
\int h(t) d m(t)>\frac{1}{2} \int f(t) d m(t)
$$

the latter being a consequence of the Monotone Convergence Theorem. The positive measure $\nu^{\prime}=h \cdot m$ is then such that $0 \leq \nu^{\prime} \leq \nu-\lambda \cdot \nu$ and $\nu^{\prime}(H)>$ $\frac{1}{2}(\nu-\lambda \cdot \nu)(H)$. Since $\left(\alpha^{*}\right)^{n}(\nu)$ has its support in a compact set which converges to the identity as $n \rightarrow \infty, h *\left(\alpha^{*}\right)^{n}(\nu)$ converges uniformly to $h$ and for large enough $n$ its support is a compact subset of $U$. Fix $\beta \in(0,1)$ so that $\int \beta \cdot h(t) d m(t)>\frac{1}{2} \int f(t) d m(t)$. Then, in view of the preceding remarks, it is possible to choose $n$ sufficiently large so that $(\beta \cdot h) \star\left(\alpha^{*}\right)^{n}(\nu) \leq \nu-\lambda \star \nu$. Now let $\lambda^{\prime}=\lambda+\beta \cdot \nu^{\prime} \star \delta(n, 0)$. Evidently $\lambda^{\prime} \geq \lambda$,

$$
\lambda^{\prime} \cdot \nu=\lambda \cdot \nu+\beta \nu^{\prime} \star\left(\alpha^{*}\right)^{n}(\nu) \leq \nu
$$

and

$$
\left(\lambda^{\prime} \cdot \nu\right)(H)=\beta \nu^{\prime} \star\left(\alpha^{*}\right)^{n}(\nu)(H)+\lambda \cdot \nu(H) \geq \beta \nu^{\prime}(H)+\lambda \cdot \nu(H)>\left(1-\frac{\delta}{2}\right) .
$$

Finally, we observe that the Radon-Nikodym derivative of $\lambda^{\prime} \cdot \nu$ is continuous because those of $\lambda \cdot \nu$ and $\nu^{\prime}$ are.

\section{THE EXAMPLE}

We use as our abelian group a subgroup of the doubly infinite product of copies of $\mathbf{Z}(2)=\{-1,+1\}$ :

$$
H=\left\{\mathbf{h}=\left(h_{n}\right)_{-\infty}^{\infty}: h_{n}= \pm 1, h_{n}=1 \text { for } n \text { sufficiently large }\right\} .
$$

Write $H_{N}$ for the subgroup of those members $\mathbf{h}$ of $H$ for which $h_{n}=1$ for all $n \geq N$. The group $H$ is topologised so that each $H_{N}$ has the product topology as a product of $\mathbf{Z}(2)$ 's. It follows that they are all compact and $H$ is locally compact. Note that $H_{N} \subset H_{N+1}$ and that $H=\bigcup_{N \in \mathbf{Z}} H_{N}$. 
The automorphism $\alpha$ is chosen to be the shift defined by $\alpha(\mathbf{h})_{n}=h_{n+1}$ $(n \in \mathbf{Z})$. It follows that $\alpha\left(H_{N}\right)=H_{N-1}$. The property (1) we require for $\alpha$ is clearly satisfied. We shall define $\mu$ to be a Riesz product measure on the subgroup $H_{0}$ (cf. [3, Chapter 7]). To define $\mu$ let $\chi_{n}$ be the character of $H$ given by $\chi(\mathbf{h})=h_{-n}$ and let $m_{N}$ be normalised Haar measure on $H_{N}$. Now $\mu$ is the weak-* limit of the sequence $\mu_{r}$ where

$$
\mu_{r}=\prod_{n=0}^{r}\left(1+a_{n} \chi_{n}\right) \cdot m_{0}
$$

and $\left(a_{n}\right)$ is a sequence of real numbers to be chosen later subject to the constraint that $\left|a_{n}\right| \leq 1$ for all $n$. We write

$$
\mu=\prod_{n=0}^{\infty}\left(1+a_{n} \chi_{n}\right) \cdot m_{0}
$$

to specify this measure even though for some choices of the sequence $\left(a_{n}\right)$ it will be singular with respect to $m_{0}$.

Alternatively, $\mu$ may be seen as an infinite product measure

$$
\mu=\star_{n=0}^{\infty}\left(\frac{\left(1+a_{n}\right)}{2} \delta(1)+\frac{\left(1-a_{n}\right)}{2} \delta\left(\mathbf{e}_{n}\right)\right)
$$

where $\left(\mathbf{e}_{n}\right)_{k}=-1$ when $n=-k$ and 1 otherwise. This may be checked by calculating the Fourier transform of $\mu$. The infinite product picture allows us to see immediately that

$$
\left(\alpha^{*}\right)^{k}(\mu)=\star_{n=0}^{\infty}\left(\frac{\left(1+a_{n}\right)}{2} \delta(1)+\frac{\left(1-a_{n}\right)}{2} \delta\left(\mathbf{e}_{n+k}\right)\right)
$$

and back in the Riesz product formalism

$$
\left(\alpha^{*}\right)^{k}(\mu)=\prod_{n=0}^{\infty}\left(1+a_{n} \chi_{n+k}\right) \cdot m_{-k} .
$$

Noting that, for $k>0, m_{-k}=\prod_{n=0}^{k-1}\left(1+\chi_{n}\right) \cdot m_{0}$, we obtain

$$
\left(\alpha^{*}\right)^{k}(\mu)=\prod_{n=0}^{\infty}\left(1+a_{n-k} \chi_{n}\right) \cdot m_{0}
$$

where $a_{n}$ is defined to be 1 for $n<0$. It is now possible to calculate $\nu$ which as we have seen is, in this context, $\star_{k=0}^{\infty}\left(\alpha^{*}\right)^{k}(\mu)$. Thus we have

$$
\nu=\prod_{n=0}^{\infty}\left(1+b_{n} \chi_{n}\right) \cdot m_{0}
$$

where $b_{n}=\prod_{k=0}^{\infty} a_{n-k}=a_{n} a_{n-1} \cdots a_{1} a_{0}$.

A straightforward generalisation of Zygmund's theorem (see [3, Theorem 7.2.2]) shows that a Riesz product $\tau=\prod_{n=0}^{\infty}\left(1+c_{n} \chi_{n}\right) \cdot m_{0}\left(\left|c_{n}\right|<1\right)$ is absolutely continuous with respect to $m_{0}$ if and only if $\sum_{n=0}^{\infty} c_{n}^{2}<\infty$. It is 
possible to give a corresponding characterisation of Riesz products which are lower semicontinuous. We note that with relatively minor changes this last result is effectively the same as ([1, Theorem 2]); the proof given here is somewhat different.

Proposition 4. The Riesz product $\tau=\prod_{n=0}^{\infty}\left(1+c_{n} \chi_{n}\right) \cdot m_{0}$ is lower semicontinuous if and only if $\sum_{n=0}^{\infty}\left|c_{n}\right|<\infty$, and in this case its Radon-Nikodym derivative has an absolutely convergent Fourier series as a function on $H_{0}$.

Proof. If $\sum_{n=0}^{\infty}\left|c_{n}\right|<\infty$ then the infinite product $\prod_{n=0}^{\infty}\left(1+c_{n} \chi_{n}\right)$ converges uniformly to a function whose Fourier series converges absolutely and so the sufficiency of the condition is clear.

For the converse first note that, since $\tau$ is absolutely continuous with respect to $m_{0}, \sum_{n=0}^{\infty} c_{n}^{2}<\infty$, so that the series

$$
f(\mathbf{h})=\sum_{n=0}^{\infty} \log \left(1+c_{n} \chi_{n}(\mathbf{h})\right)
$$

converges almost everywhere with respect to $m_{0}$. To see this, observe that the sequence $\left(\chi_{n}\right)$ consists of mutually independent random variables with respect to $m_{0}$, so that the claim is an immediate consequence of the Three Series Theorem ([2, Chapter III, Theorem 2.5]). Moreover, the function $f$ is the logarithm of the Radon-Nikodym derivative of $\tau$ with respect to $m_{0}$. The zero-one law shows that the $m_{0}$-measure of the set where $f$ equals $-\infty$ has measure 0 or 1 . In the latter case, $\tau=0$, which is absurd. In the former case, since $f$ is almost everywhere lower semicontinuous, it has an essential lower bound. In view of the fact that $\sum_{n=0}^{\infty} c_{n}^{2}<\infty$, it is straightforward to see that this is the case if and only if $\sum c_{n} \chi_{n}$ is bounded below. Now the transformation on $H_{0}$ which takes $\left(h_{n}\right)$ to $\left(-h_{n}\right)$ is continuous and so clearly takes $\tau$ to a measure $\tau^{\prime}$ which is also lower semicontinuous. But this measure is $\tau^{\prime}=\prod_{n=0}^{\infty}\left(1-c_{n} \chi_{n}\right) \cdot m_{0}$, so that by repeating the above argument, we obtain that $\sum c_{n} \chi_{n}$ is bounded. Now another application of the Three Series Theorem yields that $\sum\left|c_{n}\right|<\infty$ (loc. cit.).

To complete the description of the example we only need to find an appropriate choice for the sequence $\left(a_{n}\right)$ for which the sequence $\left(b_{n}\right)$ is square summable but not summable. This is achieved by letting $a_{n}=n / n+1 \quad(n>0)$ and $a_{0}=1$. Then $b_{n}=(n+1)^{-1}$ and we have the required example.

\section{REFERENCES}

1. Gavin Brown and William Moran, Products of random variables and Kakutani's criterion for the orthogonality of product measures, J. London Math. Soc. (2) 10 (1975), 401-405.

2. J. L. Doob, Stochastic Processes, Wiley, New York.

3. Colin C. Graham and O. Carruth McGehee, Essays in commutative harmonic analysis, Springer-Verlag, New York, 1979. 
4. V. A. Kaimanovich and A. M. Vershik, Random walks on discrete groups, Ann. Probab. 11 (1983), 457-490.

5. George A. Willis, Probability measures on groups and some related ideals in group algebras, J. Funct. Anal. 92 (1990), 202-263.

Department of Pure Mathematics, The University of Adelaide, Adelaide 5001, South Australia

Department of Mathematics, Institute of Advanced Studies, Australian National University, G.P.O. BoX 4, CANBerRa, ACT, 2601, Australia 